\title{
Estimation of traffic accident costs: a prompted model
}

\begin{abstract}
Traffic accidents are the reason for $25 \%$ of unnatural deaths in Iran. The main objective of this study is to find a simple model for the estimation of economic costs especially in Islamic countries (like Iran) in a straightforward manner. The model can show the magnitude of traffic accident costs with monetary equivalent. Data were collected from different sources that included traffic police records, insurance companies and hospitals. The conceptual framework, in our study, was based on the method of Ayati. He used this method for the estimation of economic costs in Iran. We promoted his method via minimum variables. Our final model has only three available variables which can be taken from insurance companies and police records. The running model showed that the traffic accident costs were US $\$ 2.2$ million in 2007 for our case study route.
\end{abstract}

Keyword: Model; Estimation; Traffic accident costs; Iran 